\title{
GATA-dependent regulation of TPO-induced $c$-mpl Gene Expression during Megakaryopoiesis
}

\author{
By \\ Masataka SUNOHARA ${ }^{1,2}$, Shigeru MORIKAWA ${ }^{3}$, Akira FUSE ${ }^{2}$, Iwao SATO ${ }^{1}$ \\ ${ }^{1}$ Department of Anatomy, School of Life Dentistry at Tokyo, The Nippon Dental University, \\ 1-9-20 Fujimi, Chiyoda-ku, Tokyo 102-8159, Japan \\ ${ }^{2}$ Department of Safety Research on Blood Products and Biologics, National Institute of Infectious Diseases, \\ 4-7-1 Gakuen, Musashimurayama, Tokyo 208-0011, Japan \\ ${ }^{3}$ Department of Veterinary Science, National Institute of Infectious Diseases, \\ 1-23-1 Toyama, Shinjuku-ku, Tokyo 162-8640, Japan
}

-Received for Publication, January 7, 2014-

Key Words: thrombopoietin (TPO), $c-m p l$, protein kinase C (PKC), megakaryocyte, CMK

\begin{abstract}
Summary: Thrombopoietin (TPO) and its receptor, c-Mpl, play the crucial role during megakaryocytopoiesis. Previously, we have shown that the promoter activity of $c$-mpl induced by TPO is modulated by transcription through a PKC-dependent pathway and that GATA(-77) is involved as a positive regulatory element in TPO-induced $c$-mpl gene expression in the megakaryoblastic CMK cells. In this research, to examine participating possibility of GATA promoter element in TPOinduced $c-m p l$ gene expression through a PKC-independent pathway, the promoter activity of site-directed mutagenesis and the effect of potein kinase $C$ modulator were measured by a transient transfection assay system. Together with our previous results on the TPO-induced $c$-mpl promoter, this study indicates destruction of -77GATA in $c$-mpl promoter decreased the activity by $47.3 \%$ under existence of GF109203. These results suggest that GATA promoter element plays significant role in TPO-induced $c$-mpl gene expression through a PKC-independent pathway.
\end{abstract}

\section{Introduction}

c-Mpl and its ligand, Thrombopoietin (TPO), are required for megakaryopoiesis and platelet development (Lok et al., 19941), De Sauvage et al., 19942), Wendling et al., 19943), Kaushansky ,19994)). A protein kinase C $(\mathrm{PKC})$ is a member of a family of serine/threonine protein kinases in the cytosol involved in pleiotropic processes such as cytokine secretion, cell growth and differentiation (Ohno et al., 19915), Nishizuka et al., 19926)). The human megakaryoblastic cell line, CMK, was established from the peripheral cells obtained from a patient with acute megakaryoblastic leukemia and Down' syndrome (Komatsu et al., 19897), Sato et al., 19898)). We have characterized an expression of c-Mpl on the CMK cells (Sato et al., 19898) ) and found that TPO down-regulated the expression of c-Mpl on the cell surface (Sato et al., 19989)). In the CMK cells, TPO has also been shown to activate PKC. (Hong et al., 199810)). Recently we suggested that TPO-induced $c-m p l$ promoter activity was modulated at transcription level through a PKC-dependent pathway (Sunohara et al., 200311), 200412) and that GATA(-77) is involved as a positive regulatory element in TPOinduced $\mathrm{c}-\mathrm{mpl}$ gene expression in the megakaryoblastic CMK cells(Sunohara et al., 201313)). In this research, we investigated role of GATA promoter element in TPOinduced $c-m p l$ gene expression through a PKC-independent pathway by using a transient transfection assay system.

\section{Materials and methods}

\section{Cell Culture}

The CMK cell line was established from a patient with acute megakaryoblastic leukemia and Down' syndrome (Komatsu et al., 19897), Sato et al., 19898)). As described previously (Sunohara et al., 200311), 201313), 200914), 201015), 200816)), the cells were cultured in RPMI 1640 medium (GIBCO, Grand Island, NY) containing 10\% FCS, 2mM L-glutamine, 100 units/ml penicillin, $100 \mu \mathrm{g} /$ 


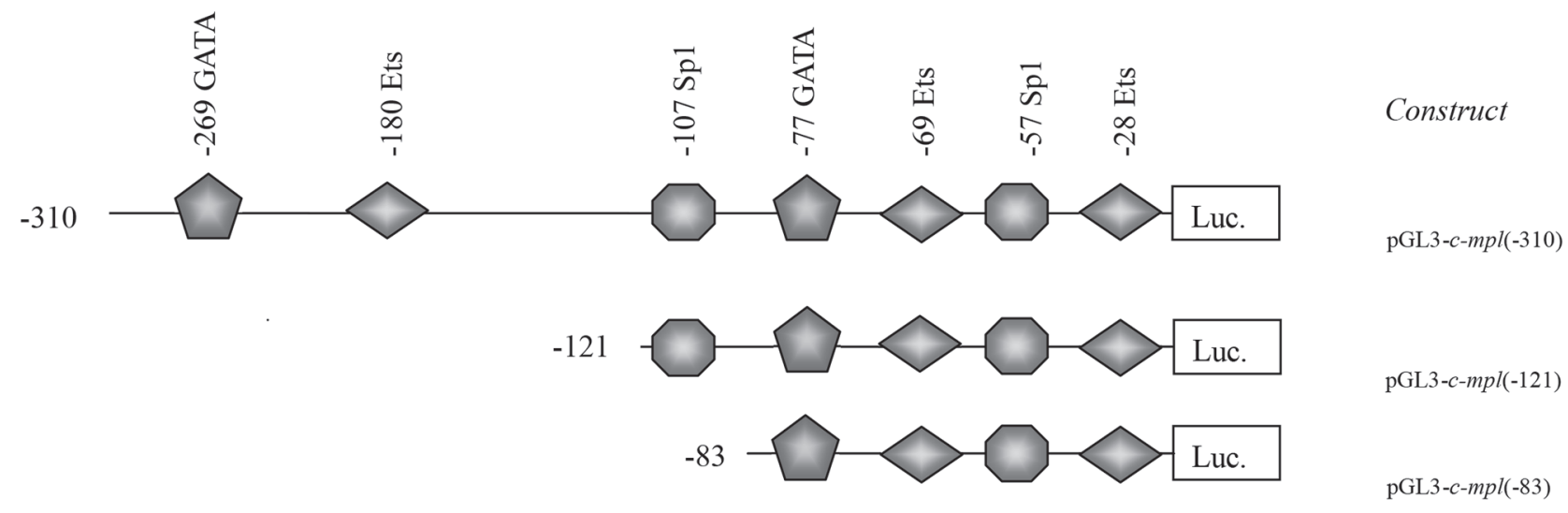

Fig. 1A. Schematic representation of putative regulatory elements in the $c-m p l$ promoter and the organization of these sites in the deletion constructs. To determine the suitable construct for hight-level specific expression, we examined the three deletion construts. Each deletion construct is represented in Fig. 1A.

$\mathrm{ml}$ streptomycin, and $0.25 \mu \mathrm{g} / \mathrm{ml}$ amphotericin B at $37^{\circ} \mathrm{C}$ in humid air containing $5 \% \mathrm{CO}_{2}$ atmosphere. $\mathrm{CMK}$ cells were cultured in RPMI 1640 medium (GIBCO, Grand Island, NY) supplemented with $10 \%$ fetal calf serum (FCS), 2mM L-glutamine, 100 units/ml penicillin, $100 \mu \mathrm{g} /$ $\mathrm{ml}$ streptomycin, and $0.25 \mu \mathrm{g} / \mathrm{ml}$ amphotericin B at $37^{\circ} \mathrm{C}$ in humidified air containing $5 \% \mathrm{CO}_{2}$ atmosphere.

\section{Reagents}

Protein kinase C modulators, GF 109203 (CALBIOCHEM Co., La Jolla, CA) was dissolved in DMSO as a stock solution and kept at $-80{ }^{\circ} \mathrm{C}$ until use. Control medium included DMSO at the highest concentration used in stock solutions did not change $c-m p l$ gene expression.

Thrombopoietin (recombinant human TPO) was purified as previously described(De Sauvage et al., 19942)).

\section{Construction of a reporter plasmid to measure the} c-mpl-promoter activity.

The construct [pGL3-c-mpl $(-310)]$ has been reported previously (Sunohara et al., 200311), 201313), 200914), $2010^{15)}$ ), the $310 \mathrm{bp}$ upstream promoter region of $c$-mpl was amplified from the chromosomal DNA of the CMK cells by polymerase chain reaction using the following primer set, (forward 5'-CTA TTC TGA TGC TAT TAT ACC ATT C-3', reverse 5'-CTT GGC TGC TGA CTT GGG C-3'). The PCR product was cloned into pGEMTeasy vector (Promega, Madison, WI) and designated as pGEM-c-mpl-prom. For the determination of the promoter activity of $c-m p l$, a $B g l$ II /Hind III fragment of pGEM-c$m p l$-prom was subcloned into a defined Bgl II /Hind III site of the pGL3-basic vector (Promega) to construct pGL3$c-m p l(-310)$ in which the luciferase gene was driven under control of the $c-m p l$ promoter (Fig.1).

\section{Promoter deletion constructs}

As described previously (Sunohara et al., 200816)), the truncated forms of the $310 \mathrm{bp}$ upstream promoter region of $c-m p l$ were amplified from the pGEM-c-mpl-prom by polymerase chain reactions using the following primer sets: c-mpl-prom (-121) (forward: 50-GGA TGG GCC CCA GA-30, reverse: 50-CTT GGC TGC TGA CTT GGG C-30), c-mpl prom (-83) (forward: 50-GGG GCT GTATCT GAC-30, reverse: 50-CTT GGC TGC TGA CTT GGG C-30). The PCR products were cloned into pGEM-Teasy vector and their sequences were confirmed. Then their Bgl II/Hind III fragments were subcloned into the Bgl II/Hind III site of the pGL3-basic vector to construct pGL3-c-mpl (-121), pGL3-c-mpl (-83), respectively (Fig. 1A). In these constructs, the luciferase gene is driven under control of the truncated forms of $c$-mpl promoter.

\section{Site-directed mutagenesis}

As reported previously (Sunohara et al., 200816)), to examine the positive regulatory elements in the $c-m p l$ promoter, we examined the effect of a of mutation within the positive regulatory motifs on the promoter activity (Fig. 2A). The promoter activity of the mutated construct (pGL3-77GATA (-)) was measured after transient transfection assay in CMK cells. The mutation destroyed the GATA sequence (GATA $\rightarrow$ GETA) in pGL3-77GATA (-) (mutated residues are underlined).

\section{Transient transfection assay}

As described previously(Sunohara et al., 200311), 201313), 200914), 201015)), three reporter plasmids, pGL3-basic vector (as a negative control), pGL3-control vector in which the luciferase gene is driven under SV40 promoter (as a positive control, Promega) and the pGL3c-mpl (-310) were used for transfection assay. A plasmid, 


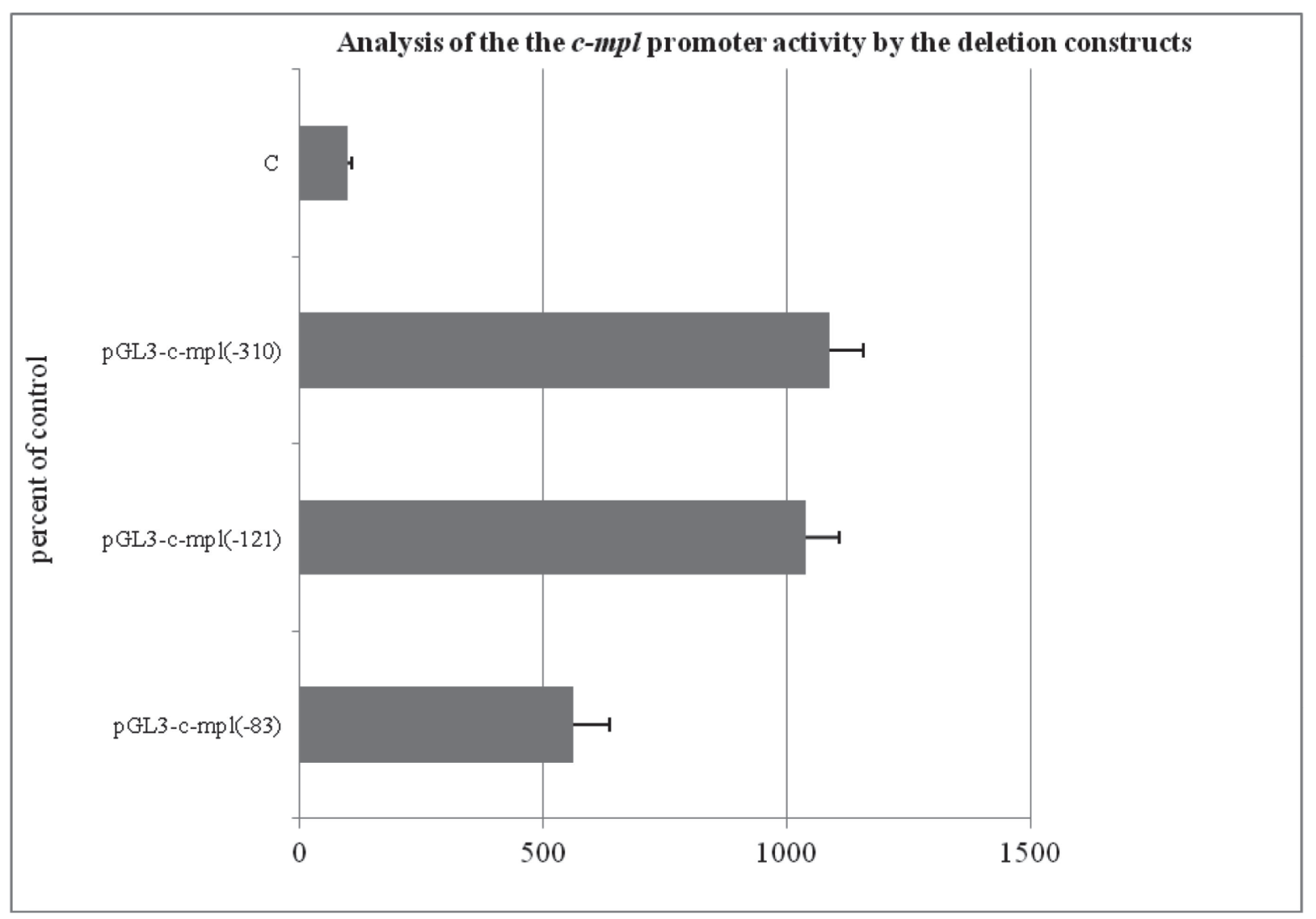

Fig. 1B. Analysis of the $c-m p l$ promoter activity by the deletion constructs. The activity of the each deletion construct (pGL3-c-mpl (-310), pGL3c-mpl (-121), pGL3-c-mpl (-83) was measured by transient transfection assay in CMK cells (Fig. 1B). Results are expressed as the percent of the control values. All experiments were performed at least three times, and results represent means $\pm \mathrm{SD}$ of at least 3 measurements.

pRL-CMV (TOYO INC MFG CO., LTD., Tokyo), which expresses Renilla (SeaPansy) luciferase under control of CMV early promoter, was used to normalize the transfection efficiency. TPO $(100 \mathrm{ng} / \mathrm{ml})$ was added to the CMK cells and then the cells were washed twice in PBS. The reporter plasmids $(1 \mu \mathrm{g})$ were co-transfected with the pRL-CMV into the CMK cells for $5 \mathrm{~h}$ by a lipofection method using the DMRIE-C reagent (GIBCO, Grand Island, NY) according to the manufacturer's instructions. Then, the cells were cultured in the growth medium (RPMI1640, 10\% FCS) for $19 \mathrm{~h}$, washed twice in PBS, lyzed in a cell lysis reagent, LCB (TOYO INC MFG CO., LTD., Tokyo) and stored at $-80{ }^{\circ} \mathrm{C}$ until luciferase assay. The luciferase activity was quantified by using a PicaGene-Dual-SeaPansy Luminescence Kit (TOYO INC MFG CO., LTD., Tokyo). $20 \mu 1$ aliquot of cell lysates were added to $100 \mu \mathrm{l}$ of luciferase reaction mixture supplied in the kit, and luciferase activity was measured with a luminometer in accordance with the manufacturer's instructions. The cell lysate of the cells transfected with a promoter-less control vector, pGL3-Basic Vector, was used as a negative control and the SeaPansy luciferase activity expressed from pRL-CMV was used to evaluate transfection efficiency and to normalize luciferase activity in all lysates. Results are expressed as the percentage of the control values. All the experiments were performed at least three times, and results represent means \pm SD of at least 3 measurements.

\section{Results}

We examined the effect of TPO on $c$-mpl promoter activity by using transient transfection assay system. Besides pGL3-c-mpl (-310), we also examined the effect of site-directed mutagenesis and PKC inhibitor (GF109303X) on TPO-induced c-mpl promoter activity.

Effect of TPO and protein kinase C inhibitor on the activation of the c-mpl promoter

After pretreatment with TPO (100 ng/ml for $3 \mathrm{~h})$, TPO increased the $c-m p l$ promoter activity and PKC inhibitor (GF109303X) reduced the basal level of the $c$ - $m p l$ promoter activity. As reported previously (Sunohara et al., 200311), 2008 ${ }^{16)}$ ), we confirmed again that PKC plays the key role in maintaining $c-m p l$ transcription in CMK cells(data not 


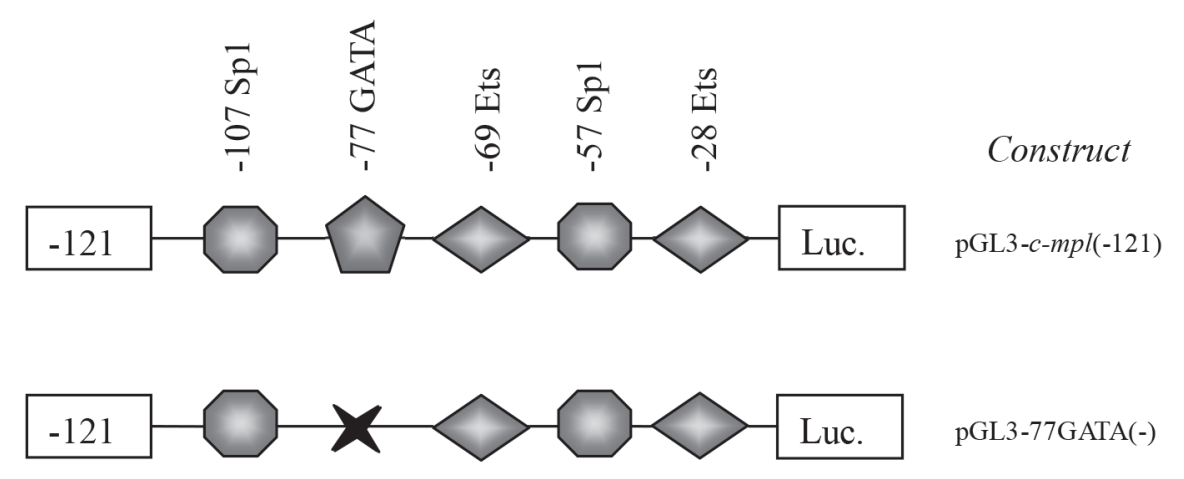

Fig. 2A. Schematic representation of the organization of putative regulatory elements in the mutated construct. Schematic representation of putative regulatory elements in pGL3-c-mpl (121) and the organization of these sites in the mutated constructs. To examine the positive regulatory elments in the $c-m p l$ promoter, we examined the effect of mutation within the positive regulatory motif on the promoter activity. The mutation within the positive regulatory motif (-77GATA) is represented in. Fig. 2A. The crosses (X) designate the mutated regions.

shown).

Effect of deletions in regulatory elements on c-mpl promoter activity

As previously described (Sunohara et al., 201313), the deletion constructs, the activity was clearly reduced from pGL3-c-mpl (-83) and we confirmed that pGL3-c-mpl $(-121)$ is the most suitable construct, for the following our experiments(Fig. 1A, 1B).

Effect of point mutations in pGL3-c-mpl(-121) on the c-mpl promoter activity

We already reported the effect of the mutation within the positive regulatory motifs on the promoter activity to examine the positive regulatory elements in the $c-m p l$ promoter. The promoter activity of the mutated construct (pGL3-77GATA) was measured after transient transfection assay in CMK cells. Destruction of -77GATA in $c$-mpl promoter decreased the activity by $22.8 \%$ ( Sunohara et al., 201313)). In this study, destruction of-77GATA motif in $c$ - $m p l$ promoter decreased its activity by $21.66 \%$ and we confirmed again $\operatorname{GATA}(-77)$ is involved as a positive regulatory element in TPO-induced $c$-mpl gene expression in CMK cells..

Effect of GF109203 and mutation within the positive regulatory motif on TPO-induced c-mpl promoter activity

In this research, to examine participating possibility of GATA promoter element in TPO-induced $c$-mpl gene expression through a PKC-independent pathway, CMK cells were cultured with GF109203 and then the promoter activity of site-directed mutagenesis and the effect of potein kinase $\mathrm{C}$ modulator were measured by a transient transfection assay system.

As shown in Fig. 2B, destruction of -77GATA in $c-m p l$ promoter decreased the activity by $47.3 \%$ under existens of GF109203. Take this results into consideration,
GATA promoter element play significant roles in TPO-induced $c-m p l$ gene expression through a PKC-independent pathway.

\section{Discussion}

It was recently reported that the promoter activity of $c-m p l$ was upregulated by TPO pretreatment and TPO-induced $c-m p l$ promoter activity was modulated at transcription level through a PKC-dependent pathway in the CMK cells (Sunohara et al., 200311), 200412)). In this research, we investigated the role of GATA promoter element in TPO-induced $c$ - $m p l$ gene expression through a PKC-independent pathway by using a transient transfection assay system.

The data of the promoter activity of the deletion constructs upon TPO treatment clearly showed that the important positive regulatory elements appeared to be at position less than -121 in $c$ - $m p l$ promoter. So we confirmed that pGL3-c-mpl $(-121)$ is the most suitable construct for the following our experiments (Fig. 2B).

To examine the positive regulatory elements in the $c-m p l$ promoter, the promoter activity of the mutated construct (pGL3-77GATA) was measured after transient transfection assay in CMK cells and destruction of -77GATA in c-mpl promoter decreased the activity by $22.8 \%$ (Fig. 3B).

The glycoprotein IIb promoter contains a specific erythromegakaryocytic enhancer that may interact with GATA-1 in megakaryocytic cells (Prandini et al., 199217)) and also GATA and Ets recognition sequences are an important determinant of megakaryocytic gene expression (Lemarchandel et al., 199318)). On the other hans, GATA1 -induced upregulation of $c-m p l$ is involved in erythroid and megakaryocytic differentiation in the murine myeloid cell line (Yamaguchi et al., 199819) and GATA-1 serves multiple functions during megakaryocyte development 




Fig. 2B. Effect of GF109203 and mutation within the positive regulatory motif on TPO-induced c-mpl promoter activity. The promoter activity of the mutated construct [pGL3-77GATA(-)] was measured by transient transfection assay in CMK cells (Fig. 2B). CMK cells were pretreated with TPO $(100 \mathrm{ng} / \mathrm{ml}$ for $3 \mathrm{hr}$ ), washed twice and transfected with $c$ - $m p l$-prom-luc for $5 \mathrm{~h}$ and then incubated in the complete medium for $19 \mathrm{~h}$. Protein kinase C inhibitor, GF109203 $(10 \mu \mathrm{M})$ was added to CMK cells from $1 \mathrm{~h}$ before TPO pretreatment. Then the cells were washed twice and transfected with $c$-mpl-luc for $5 \mathrm{~h}$. After incubation in complete medium for $19 \mathrm{~h}$, the c-mpl promoter activity was measured and results are expressed as the percent of the control values. All the experiments were performed at least three times, and results represent means \pm SD of at least 3 measurements.

(Vyas et al., 199920)).

As reported before, our research clearly defines that -77GATA was a positive regulatory element and involved in $c$-mpl gene expression induced by TPO.

Together with our previous results, this study suggests clearly that destruction of -77GATA in $c-m p l$ promoter decreased the activity by $47.3 \%$ under existence of GF109203. These results are shown that GATA promoter element plays significant role in TPO-induced $c$ - $m p l$ gene expression through a PKC-independent pathway.

Further studies are needed to elucidate the PKC-independent modulation mechanism of $c$ - $m p l$ gene expression induced by TPO in human megakaryocytic CMK cells.

\section{Acknowledgments}

This work was supported by a grant from the Ministry of Education, Science Sports and Culture of Japan (No. 10771001) and by a Grant-in-Aid for the Encouragement of Young Scientists (No. 13771085) from the Japan Society for the Promotion of Science (JSPS).

\section{References}

1) Lok S, Kaushansky K, Holly RD, Kuijper JL, Lofton-Day CE, Oort PJ, Grant FJ, Heipel MD, Burkhead SK, Kramer JM, et al: Cloning and expression of murine thrombopoietin cDNA and stimulation of platelet production in vivo. Nature 1994; 369:519-20.

2) De Sauvage FJ, Hass PE, Spencer SD, Malloy BE, Gurney AL, Spencer SA, Darbonne WC, Henzel WJ, Wong SC, Kuang WJ, et al: Stimulation of megakaryocytopoiesis and thrombopoiesis by the c-Mpl ligand. Nature 1994; 369:533-8.

3) Wendling F, Maraskovsky E, Debili N, Florindo C, Teepe M, Titeux M, Methia N, Breton-Gorius J, Cosman D, Vainchenker W: c-Mpl ligand is a humoral regulator of megakaryocytopoiesis. Nature 1994; 369:571-4.

4) Kaushansky K: The enigmatic megakaryocyte gradually reveals its secrets. Bioessays 1999; 21:353-60.

5) Ohno S, Akita Y, Hata A, Osada S, Kubo K, Konno Y, Akimoto K, Mizuno K, Saido T, Kuroki T, et al: Structural and functional diversities of a family of signal transducing protein kinases, protein kinase C family: two distinct classes of PKC, conventional cPKC and novel nPKC. Adv Enzyme Regul 1991; 31:287-303.

6) Nishizuka Y: Intracellular signaling by hydrolysis of phospholipids and activation of protein kinase C. Science 1992; 258:607-14.

7) Komatsu N, Suda T, Moroi M, Tokuyama N, Sakata Y, Okada M, Nishida T, Hirai Y, Sato T, Fuse A, et al: Growth and differentiation of a human megakaryoblastic cell line CMK. Blood 1989; 74:42-8. 
8) Sato T, Fuse A, Eguchi M, Hayashi Y, Ryo R, Adachi M, Kishimoto Y, Teramura M, Mizoguchi H, Shima Y, et al: Establishment of a human leukaemic cell line (CMK) with megakaryocytic characteristics from a Down's syndrome patient with acute megakaryoblastic leukemia. Br J Haematol 1989; 72:184-90.

9) Sato T, Fuse A, Niimi H, Fielder PJ, Avraham H: Binding and regulation of thrombopoietin to human megakaryocytes. Br J Haematol 1998; 100:704-11.

10) Hong Y, Dumenil D, van der Loo B, Goncalves F, Vainchenker W, Erusalimsky JD: Protein kinase $\mathrm{C}$ mediates the mitogenic action of thrombopoietin in c-Mpl-expressing UT-7 cells. Blood 1998; 91(3):813-22.

11) Sunohara M, Morikawa S, Sato T, Sato I, Sato T, Fuse A: Modulation of human $c$ - $m p l$ gene expression by thrombopoietin through protein kinase C. Cell Mol Biol 2003; 49. Online Pub: OL393-8.

12) Sunohara M, Sato I, Sato T, Fuse A: Modulation mechanism of c-mpl gene expression in human megakaryoblastic CMK cells. Dentistry in Japan 2004; 40:3-6.

13) Sunohara M, Morikawa S, Fuse A, Sato I. : Role of promoter element in c-mpl gene expression induced by TPO. Okajimas Folia Anat Jpn. 2013; 89(4):131-5.

14) Sunohara M, Morikawa S, Murata H, Fuse A, Sato I:Modulation mechanism of c-Mpl promoter activity in megakaryoblastic cells Okajimas Folia Anat Jpn. 2009 Nov; 86(3):89-91.

15) Sunohara M, Morikawa S, Murata H, Fuse A, Sato I: PKC plays a crucial roles in c-mpl gene expression in megakaryoblastic cells. Okajimas Folia Anat Jpn. 2010 Nov; 87(3):151-4.

16) Sunohara M, Morikawa S, Sato T, Miyado M, Sato I, Sato T, Fuse A. Promoter regulatory motifs involved in $c$ - $m p l$ gene expression induced by PMA. Cell Biol Int.2008; 32(6):692-7.

17) Prandini MH, Uzan G, Martin F, Thevenon D, Marguerie G. Characterization of a specific erythromegakaryocytic enhancer within the glycoprotein IIb promoter. J Biol Chem 1992; 267:10370e4.

18) Lemarchandel V, Ghysdael J, Mignotte V, Rahuel C, Romeo PH. GATA and Ets cis-acting sequences mediate megakaryocyte-specific expression. Mol Cell Biol 1993; 13:668e76.

19) Yamaguchi Y, Zon LI, Ackerman SJ, Yamamoto M, Suda T. Forced GATA-1 expression in the murine myeloid cell line M1: induction of c-Mpl expression and megakaryocytic/erythroid differentiation. Blood 1998; 91:450e7.

20) Vyas P, Ault K, Jackson CW, Orkin SH, Shivdasani RA. Consequences of GATA-1 deficiency in megakaryocytes and platelets Blood 1999; 93:2867e75. 